\title{
On Suzuki and Wardowski-Type Contraction Multivalued Mappings in Partial Symmetric Spaces
}

\author{
Mohammad Asim, ${ }^{1}$ Hassen Aydi $\left(\mathbb{D},{ }^{2,3,4}\right.$ and Mohammad Imdad ${ }^{5}$ \\ ${ }^{1}$ Department of Mathematics, Faculty of Science, Shree Guru Gobind Singh Tricentenary University, Gurugram, Haryana, India \\ ${ }^{2}$ Université de Sousse, Institut Supérieur d'Informatique et des Techniques de Communication, H. Sousse, 4000 Sousse, Tunisia \\ ${ }^{3}$ Department of Mathematics and Applied Mathematics, Sefako Makgatho Health Sciences University, Ga-Rankuwa, South Africa \\ ${ }^{4}$ China Medical University Hospital, China Medical University, Taichung, 40402, Taiwan \\ ${ }^{5}$ Department of Mathematics, Aligarh Muslim University, Aligarh 202002, India
}

Correspondence should be addressed to Hassen Aydi; hassen.aydi@isima.rnu.tn

Received 7 November 2020; Accepted 21 May 2021; Published 11 June 2021

Academic Editor: Yoshihiro Sawano

Copyright (c) 2021 Mohammad Asim et al. This is an open access article distributed under the Creative Commons Attribution License, which permits unrestricted use, distribution, and reproduction in any medium, provided the original work is properly cited.

The purpose of this paper is to provide some fixed-point results for Suzuki and Wardowski-type contraction multivalued mappings in partial symmetric spaces. We give some examples to support and substantiate the developed notions and obtained results. Also, we use one of our main results to establish the existence and uniqueness of the solution for a system of integral inclusions.

\section{Introduction}

In 1922, Banach [1] formulated a very famous, fruitful, useful, and core fixed-point result known as the Banach contraction principle on a complete metric space. This celebrated result has been generalized and extended in several directions by various authors on some generalized metric spaces such as partial metric spaces [2], partial JS-metric spaces [3], metriclike spaces [4], $b$-metric spaces [5], rectangular metric spaces [6], controlled and doubled metric spaces $[7,8]$, generalized $b$ -metric spaces [9], and extended rectangular $b$-metric spaces [10]. Sometimes, one may come across a situation wherein the full force of metric conditions is not required in the enunciation of fixed-point results. Motivated by this observation, several researchers established fixed and common fixedpoint results in symmetric spaces which did not require triangular inequality, i.e., a symmetric $\chi$ on a nonempty set $X$ is a mapping $\chi: X \times X \longrightarrow[0, \infty)$ which satisfies $d(\rho, \rho)$ $=0$ iff $\rho=\varsigma$, and $d(\rho, \varsigma)=d(\varsigma, \rho)$ for all $\rho, \varsigma \in X$.

Very recently, Asim et al. [11] introduced the class of partial symmetric spaces and proved some related fixedpoint results for single-valued and multivalued mappings. The theory of multivalued mappings plays an important role in many areas of mathematics due to its diverse appli- cations, namely in differential equations, integral equations, optimization problems, game theory, control theory, economics, etc. Aydi et al. $[12,13]$ proved fixed and common fixed-point results for multivalued maps in partial Hausdorff metric spaces to generalize fixed-point results due to Nadler [14].

In 2008, Suzuki [15] introduced a class of new types of contraction mappings and established fixed-point theorems for such mappings, which are genuine improvements of the Banach contraction principle. Thereafter, many authors attempted to extend such results for multivalued maps (employing the Pompieu-Hausdorff metric). In 2012, Wardowski [16] initiated the notion of a new kind of nonlinear contractions, namely $F$-contractions and proved some related fixed-point results. In recent years, the idea of $F$ -contractions has been generalized and improved in several ways and directions. For more details, see [17-21].

In our paper, based on Suzuki and Wardowski-type contractions, we consider multivalued mappings, and we establish some related fixed-point results in the setting of partial symmetric spaces. Some examples are also furnished to demonstrate the utility of our results. As an application of Theorem 16, we establish the existence and uniqueness of the solution for a system of integral inclusions. 


\section{Preliminaries}

In what follows, we collect some relevant definitions and auxiliary results needed in the sequel.

Definition 1 [11]. Let $X$ be a nonempty set. The function $\chi: X \times X \longrightarrow[0, \infty)$ satisfying

$\chi^{1} \chi(\rho, \varsigma)=\chi(\rho, \rho)=\chi(\varsigma, \varsigma)$ iff $\rho=\varsigma$,

$\chi^{2} \chi(\rho, \rho) \leq \chi(\rho, \varsigma)$,

$\chi^{3} \chi(\rho, \varsigma)=\chi(\varsigma, \rho)$ is said to be a partial symmetric, and the pair $(X, \chi)$ is called a partial symmetric space.

A partial symmetric space $(X, \chi)$ reduces to a symmetric space if $\chi(x, x)=0$, for all $x \in X$. Observe that every symmetric space is a partial symmetric space. The converse is not true as it is shown in the following examples.

Example 2. Let $X=\mathbb{R}$ and define $\chi: X \times X \longrightarrow[0, \infty)$ by

$$
\chi(\rho, \varsigma)=|\rho-\varsigma|^{p}+|\rho-\varsigma|^{q}+\alpha
$$

where $p, q>1$ and $\alpha \geq 0$.

Example 3. Let $X=[0, \infty)$ and define $\chi: X \times X \longrightarrow[0, \infty)$ by

$$
\chi(\rho, \varsigma)=(\max \{\rho, \varsigma\})^{p}+|\rho-\varsigma|^{q},
$$

where $p, q>1$.

Example 4. Let $X=[0, \pi)$ and define $\chi: X \times X \longrightarrow[0, \infty)$ by

$$
\chi(\rho, \varsigma)=\sin |\rho-\varsigma|+\alpha
$$

where $\alpha \geq 0$.

Example 5. Let $X=[0, \pi)$ and define $\chi: X \times X \longrightarrow[0, \infty)$ by

$$
\chi(\rho, \varsigma)=(\max \{\rho, \varsigma\})^{p}+e^{|\rho-\varsigma|},
$$

where $p>1$.

Let $(X, \chi)$ be a partial symmetric space. The $\chi$-open ball with a center $\rho \in X$ and a radius $\varepsilon>0$ is defined by

$$
B_{\chi}(\rho, \varepsilon)=\{\varsigma \in \rho: \chi(\rho, \varsigma)<\chi(\rho, \rho)+\varepsilon\} .
$$

Similarly, the $\chi$-closed ball with a center $\rho \in X$ and a radius $\varepsilon>0$ is defined by

$$
B_{\chi}[\rho, \varepsilon]=\{\varsigma \in X: \chi(\rho, \varsigma) \leq \chi(\rho, \rho)+\varepsilon\}
$$

The family of $\chi$-open balls denoted by

$$
\mathcal{U}_{\chi}=\left\{B_{\chi}(\rho, \varepsilon): \rho \in X, \varepsilon>0\right\}
$$

forms on $X$ a basis of some topology $\tau_{\chi}$.
Lemma 6. Let $\left(X, \tau_{\chi}\right)$ be a topological space and $f: X \longrightarrow X$ be given mapping. If $f$ is continuous; then, for every convergent sequence $\left\{\rho_{n}\right\}$ to a point $\rho$ in $X$, the sequence $\left\{f \rho_{n}\right\}$ converges to $f \rho$. The converse holds if $X$ is metrizable.

We require some more definitions, namely, a convergent sequence, a Cauchy sequence, and a complete partial symmetric space, in our forthcoming discussions.

Definition 7 [11]. A sequence $\left\{\rho_{n}\right\}$ in $(X, \chi) \chi$-converges to $\rho \in X$, with respect to $\tau_{\chi}$, if

$$
\chi(\rho, \rho)=\lim _{n \longrightarrow \infty} \chi\left(\rho_{n}, \rho\right) .
$$

Definition 8 [11]. A sequence $\left\{\rho_{n}\right\}$ in $(X, \chi)$ is $\chi$-Cauchy iff $\lim _{n, m \rightarrow \infty} \chi\left(\rho_{n}, \rho_{m}\right)$ exists and is finite.

Definition 9 [11]. A partial symmetric space $(X, \chi)$ is $\chi$-complete if each $\chi$-Cauchy sequence $\left\{\rho_{n}\right\}$ in $X$ is $\chi$-convergent with respect to $\tau_{\chi}$ to a point $\rho \in X$ such that

$$
\chi(\rho, \rho)=\lim _{n \longrightarrow \infty} \chi\left(\rho_{n}, \rho\right)=\lim _{n, m \longrightarrow \infty} \chi\left(\rho_{n}, \rho_{m}\right) .
$$

Let $(X, \chi)$ be a partial symmetric space and $\mathscr{C} \mathscr{B}^{\chi}(X)$ be the set of all nonempty, $\chi$-closed, and bounded subsets of $(X, \chi)$. Moreover, for $\mathscr{L}, \mathscr{M} \in \mathscr{C}_{\mathscr{B}}^{\chi}(X)$ and $\rho \in X$, we define

$$
\begin{aligned}
& \operatorname{dist}_{\chi}(\rho, \mathscr{L})=\inf \{\chi(\rho, l): l \in \mathscr{L}\}, \\
& \delta_{\chi}(\mathscr{L}, \mathscr{M})=\sup \left\{\operatorname{dist}_{\chi}(l, \mathscr{M}): l \in \mathscr{L}\right\}, \\
& \delta_{\chi}(\mathscr{M}, \mathscr{L})=\sup \left\{\operatorname{dist}_{\chi}(m, \mathscr{L}): m \in \mathscr{M}\right\} .
\end{aligned}
$$

Let $H_{\chi}(.,$.$) be the partial Pompieu-Hausdorff symmetric,$ that is

$$
H_{\chi}(\mathscr{L}, \mathscr{M})=\max \left\{\delta_{\chi}(\mathscr{L}, \mathscr{M}), \delta_{\chi}(\mathscr{M}, \mathscr{L})\right\}
$$

\section{Main Results}

Given that $f: X \longrightarrow \mathscr{C}_{\mathscr{B}}^{\chi}(X)$, then for every $\rho \in X$, we define

$$
\mathfrak{S}(\chi, f, \rho)=\sup \left\{\chi\left(\rho_{i}, \rho_{j}\right): i, j \in \mathbb{N}\right\}
$$

where $\rho_{i} \in f \rho_{i-1}$ and $\rho_{j} \in f \rho_{j-1}$

Now, we present the following lemma proved in [11], which is needed in the sequel.

Lemma 10. Let $(X, \chi)$ be a partial symmetric space and $\mathscr{L}$, $\mathscr{M} \in \mathscr{C} \mathscr{B}^{\chi}(X)$. Then, for all $h>1$ and $l \in \mathscr{L}$, there is $m \in \mathscr{M}$ so that

$$
\chi(l, m) \leq h H_{\chi}(\mathscr{L}, \mathscr{M})
$$


Next, let $\psi:[0,1) \longrightarrow(0,1]$ be the nonincreasing function defined by

$$
\psi(r)=\left(\begin{array}{ll}
1 & \text { if } 0 \leq r<\frac{1}{2} \\
1-r & \text { if } \frac{1}{2} \leq r<1 .
\end{array}\right.
$$

Theorem 11. Let $(X, \chi)$ be a complete partial symmetric space and $f: X \longrightarrow \mathscr{C} \mathscr{B}^{\chi}(X)$.

Assume the following:

(Si) There exists $0 \leq r<1$ such that $f$ satisfies the condition

$\psi(r) \operatorname{dist}_{\chi}(\rho, f \rho) \leq \chi(\rho, \varsigma)$ implies $H_{\chi}(f \rho, f \varsigma) \leq r M(\rho, \varsigma)$,

for all $\rho, \varsigma \in X$, where $\psi$ is defined by (14) and

$M(\rho, \varsigma)=\max \left\{\chi(\rho, \varsigma), \operatorname{dist}_{\chi}(\rho, f \rho), \operatorname{dist}_{\chi}(\varsigma, f \varsigma), \operatorname{dist}_{\chi}(\rho, f \varsigma), \operatorname{dist}_{\chi}(\varsigma, f \rho)\right\}$

(Sii) There is $\rho_{0} \in X$ so that $\mathfrak{S}\left(\chi, f, \rho_{0}\right)<\infty$

(Siii) The function $\rho \longrightarrow$ dist $_{\chi}(\rho, f \rho)$ is lower semicontinuous.

Then, $f$ has a unique fixed point.

Proof. By (Si), there exists $0 \leq r<1$ such that (15) holds. Let $r_{1} \in(0,1)$ be such that $0 \leq r \leq r_{1}<1$. For such $\rho_{0} \in X$, define a sequence $\left\{\rho_{n}\right\} \in X$ by $\rho_{n+1} \in f \rho_{n}$. If we assume that $\rho_{n_{0}} \in$ $f \rho_{n_{0}}$ for some $n_{0} \in \mathbb{N}$, then $\rho_{n_{0}}$ is a fixed point of $f$, and we are done. Now, suppose that $\rho_{n} \notin f \rho_{n}$ for each $n \in \mathbb{N}$, and so $\chi\left(\rho_{n}, \rho_{n+1}\right)>0$. From Lemma 10 (with $h=1 / \sqrt{r_{1}}$ ) and condition (15) (for any arbitrary $n \in \mathbb{N}$ and for all $i, j \in \mathbb{N}$ ), we have

$$
\chi\left(\rho_{n+i}, \rho_{n+j}\right) \leq \frac{1}{\sqrt{r_{1}}} H_{\chi}\left(f \rho_{n-1+i}, f \rho_{n-1+j}\right) .
$$

Since $\psi(r) \leq 1$, we get

$\psi(r) \operatorname{dist}_{\chi}\left(\rho_{n+i}, f \rho_{n+i}\right) \leq \operatorname{dist}_{\chi}\left(\rho_{n+i}, f \rho_{n+i}\right) \leq \chi\left(\rho_{n+i}, \rho_{n+1+i}\right)$.

From (15) and (17), we have

$$
\begin{aligned}
\chi\left(\rho_{n+i}, \rho_{n+j}\right) \leq & \frac{1}{\sqrt{r_{1}}} H_{p}\left(f \rho_{n-1+i}, f \rho_{n-1+j}\right) \\
\leq & \sqrt{r_{1}} M\left(\rho_{n-1+i}, \rho_{n-1+j}\right) \\
\leq & \sqrt{r_{1}} \max \left\{\chi\left(\rho_{n-1+i}, \rho_{n-1+j}\right),\right. \\
& \operatorname{dist}_{\chi}\left(\rho_{n-1+i}, f \rho_{n-1+i}\right), \operatorname{dist}_{\chi}\left(\rho_{n-1+j}, f \rho_{n-1+j}\right), \\
& \left.\operatorname{dist}_{\chi}\left(\rho_{n-1+i}, f \rho_{n-1+j}\right), \operatorname{dist}_{\chi}\left(\rho_{n-1+j}, f \rho_{n-1+i}\right)\right\} . .
\end{aligned}
$$

The above inequality is true for all $i, j \in N$; therefore, by conditions (Sii) and (12), we have

$$
\mathfrak{s}\left(\chi, f, f^{n} \rho_{0}\right) \leq \sqrt{r_{1}} \mathfrak{S}\left(\chi, f, f^{n-1} \rho_{0}\right) .
$$

By repeating this process, we have (for all $n \geq 1$ )

$$
\mathfrak{S}\left(\chi, f, f^{n} \rho_{0}\right) \leq\left(\sqrt{r_{1}}\right)^{n} \mathfrak{S}\left(\chi, f, \rho_{0}\right) .
$$

Now

$\chi\left(\rho_{n}, \rho_{n+m}\right) \leq \mathfrak{S}\left(\chi, f, f^{n} \rho_{0}\right) \leq\left(\sqrt{r_{1}}\right)^{n} \mathfrak{S}\left(\chi, f, \rho_{0}\right), \forall n, m \in \mathbb{N}$.

Since $\mathfrak{S}\left(\chi, f, \rho_{0}\right)<\infty$ and $r_{1} \in(0,1)$, then

$$
\lim _{n, m \longrightarrow \infty} \chi\left(\rho_{n}, \rho_{n+m}\right)=0,
$$

so that $\left\{\rho_{n}\right\}$ is a $\chi$-Cauchy sequence in $X$. In view of the $\chi$ -completeness of $X$, there exists $\rho \in X$ such that $\left\{\rho_{n}\right\} \chi$-converges to $\rho$. Thus

$$
\chi(\rho, \rho)=\lim _{n \longrightarrow \infty} \chi\left(\rho_{n}, \rho\right)=\lim _{n, m \longrightarrow \infty} \chi\left(\rho_{n}, \rho_{n+m}\right)=0 .
$$

Assume that $\rho \longrightarrow \operatorname{dist}_{\chi}(\rho, f \rho)$ is lower semicontinuous. Then

$$
\operatorname{dist}_{\chi}(\rho, f \rho) \leq \liminf _{n \longrightarrow \infty} \operatorname{dist}_{\chi}\left(\rho_{n}, f \rho_{n}\right) \leq \lim _{n \longrightarrow \infty} \chi\left(\rho_{n}, \rho_{n+1}\right)=0 .
$$

Therefore, $\operatorname{dist}_{\chi}(\rho, f \rho)=0$, that is, $\rho \in f \rho$. Hence, $\rho$ is a fixed point of $f$.

Next, let us show that $f$ has a unique fixed point. Suppose there are $\rho, \varsigma \in X$ such that $\rho \in f \rho$ and $\varsigma \in f \varsigma$. Then, by conditions $(S i)$ and $(\chi 2)$, we have

$$
\psi(r) \operatorname{dist}_{\chi}(\rho, f \rho) \leq \operatorname{dist}_{\chi}(\rho, f \rho) \leq \chi(\rho, \rho) \leq \chi(\rho, \varsigma),
$$

which implies (by Lemma 10 with $h=1 / \sqrt{r_{1}}$ and $0 \leq r$ $\left.\leq r_{1}<1\right)$

$$
\begin{aligned}
\chi(\rho, \varsigma) \leq & \frac{1}{\sqrt{r_{1}}} H_{\chi}(f \rho, f \varsigma) \leq \sqrt{r_{1}} M((\rho, \varsigma)) \\
= & \sqrt{r_{1}} \max \left\{\chi(\rho, \varsigma), \operatorname{dist}_{\chi}(\rho, f \rho), \operatorname{dist}_{\chi}(\varsigma, f \varsigma),\right. \\
& \left.\operatorname{dist}_{\chi}(\rho, f \varsigma), \operatorname{dist}_{\chi}(\rho, f \varsigma)\right\} \\
\leq & \sqrt{r_{1}} \max \{\chi(\rho, \varsigma), \chi(\rho, \rho), \chi(\varsigma, \varsigma), \chi(\rho, \varsigma), \chi(\varsigma, \rho)\} \\
= & \sqrt{r_{1}} \chi(\rho, \varsigma)<\chi(\rho, \varsigma),
\end{aligned}
$$

a contradiction, so that $\chi(\rho, \varsigma)=0$, which implies that $\rho=\varsigma$. Hence, $f$ has a unique fixed point. This completes the proof. 
Example 12. Take $X=\{0,1 / 10,1 / 5\}$. The partial symmetric $\chi: X \times X \longrightarrow[0, \infty)$ is defined by

$$
\chi(\rho, \varsigma)=\frac{1}{2}|\rho-\varsigma|^{2}+\frac{1}{4}(\max \{\rho, \varsigma\})^{2}, \forall \rho, \varsigma \in X .
$$

Then, $(X, \chi)$ is a $\chi$-complete symmetric space. Note that $\{0\}$ and $\{1 / 10\}$ are bounded sets in $(X, \chi)$. In fact, if $\rho \in\{0,1 / 10,1 / 5\}$, then

$\rho \in\{\overline{0}\} \Longleftrightarrow \operatorname{dist}_{\chi}(\rho,\{0\})=\chi(\rho, \rho) \Longleftrightarrow \frac{3 \rho^{2}}{4}=\frac{\rho^{2}}{4} \Longleftrightarrow \rho=0 \Longleftrightarrow \rho \in\{0\}$.

Hence, $\{0\}$ is closed. Next

$$
\begin{aligned}
\rho & \in\left\{\frac{\overline{1}}{10}\right\} \Longleftrightarrow \operatorname{dist}_{\chi}\left(\rho,\left\{\frac{1}{10}\right\}\right) \\
& =\chi(\rho, \rho) \Longleftrightarrow \min \left\{\frac{3 \rho^{2}}{4}, \frac{1}{2}\left|\rho-\frac{1}{10}\right|^{2}+\frac{1}{4}\left(\max \left\{\rho, \frac{1}{10}\right\}\right)^{2}\right\} \\
& =\frac{\rho^{2}}{4} \Longleftrightarrow \rho \in\left\{\frac{1}{10}\right\} .
\end{aligned}
$$

Hence, $\{1 / 10\}$ is also closed. Now, define $f: X \longrightarrow \mathscr{C}$ $\mathscr{B}^{\chi}(X)$ by

$$
f 0=f \frac{1}{10}=\{0\} \text { and } f \frac{1}{5}=\left\{\frac{1}{10}\right\} .
$$

Clearly, by a routine calculation, one can easily show that $x \longrightarrow \operatorname{dist}_{\chi}(\rho, f \rho)$ is lower semicontinuous. To prove the contractive condition $(\mathrm{Si})$ of Theorem 11, we need the following.

Case 13. Let $\rho=0$. Then

$$
\psi(r) \operatorname{dist}_{\chi}(0, f 0)=0 \leq \chi(0, \varsigma) \text {, for all } \varsigma \in X .
$$

For $\varsigma=0$ or $\varsigma=1 / 10$, we have.

$$
H_{\chi}(f 0, f \varsigma)=H_{\chi}(0,0)=0 \leq r \chi(0, \varsigma) .
$$

For $\varsigma=1 / 5$, we have

$$
\begin{aligned}
H_{\chi}\left(f 0, f \frac{1}{5}\right) & =H_{\chi}\left(0, \frac{1}{10}\right)=\frac{1}{2}\left|0-\frac{1}{10}\right|^{2}+\frac{1}{4}\left(\max \left\{0, \frac{1}{10}\right\}\right)^{2} \\
& =\frac{1}{4}\left(\frac{1}{2}\left|0-\frac{1}{5}\right|^{2}+\frac{1}{4}\left(\max \left\{0, \frac{1}{5}\right\}\right)^{2}\right) \\
& =\frac{1}{4} \chi\left(0, \frac{1}{5}\right) .
\end{aligned}
$$

Case 14. Let $\rho=1 / 10$. Then, $\psi(r) \operatorname{dist}_{\chi}(1 / 10, f 1 / 10)=3 / 400$ $\leq \chi(0, \varsigma)$, for all $\varsigma \in X$.
For $\varsigma=0$ or $\varsigma=1 / 10$, we have

$$
H_{\chi}\left(f \frac{1}{10}, f \varsigma\right)=0<r \frac{3 \varsigma^{2}}{4} \leq r \chi\left(\frac{1}{10}, \varsigma\right) .
$$

For $\varsigma=1 / 5$, we have

$$
\begin{aligned}
H_{\chi}\left(f \frac{1}{10}, f \frac{1}{5}\right) & =H_{\chi}\left(\{0\},\left\{\frac{1}{10}\right\}\right) \\
& =\frac{1}{2}\left|0-\frac{1}{10}\right|^{2}+\frac{1}{4}\left(\max \left\{0, \frac{1}{10}\right\}\right)^{2} \\
& =\frac{1}{2}\left(\frac{1}{2}\left|\frac{1}{10}-\frac{1}{5}\right|^{2}+\frac{1}{4}\left(\max \left\{\frac{1}{10}, \frac{1}{5}\right\}\right)^{2}\right) \\
& =\frac{1}{2} \chi\left(\frac{1}{10}, \frac{1}{5}\right) .
\end{aligned}
$$

Case 15. Let $\rho=1 / 5$. Then

$$
\psi(r) \operatorname{dist}_{\chi}\left(\frac{1}{5}, f \frac{1}{5}\right)=\frac{3}{200} \leq \chi\left(\frac{1}{5}, \varsigma\right), \text { for } \varsigma \in X .
$$

For $\varsigma=0$, we have

$$
H_{\chi}\left(f \frac{1}{5}, f \varsigma\right)=H_{\chi}\left(\left\{\frac{1}{10}\right\},\{0\}\right) \leq \frac{1}{4} \chi\left(\frac{1}{5}, 0\right) .
$$

For $\varsigma=1 / 10$, we get

$H_{\chi}\left(f \frac{1}{5}, f \frac{1}{10}\right)=H_{\chi}\left(\left\{\frac{1}{10}\right\},\{0\}\right) \leq \frac{1}{4} \chi\left(\frac{1}{5}, 0\right)$.

For $\varsigma=1 / 5$, we have

$$
H_{\chi}\left(f \frac{1}{5}, f \frac{1}{5}\right)=H_{\chi}\left(\left\{\frac{1}{10}\right\},\left\{\frac{1}{10}\right\}\right) \leq \frac{1}{4} \chi\left(\frac{1}{5}, \frac{1}{5}\right) .
$$

Hence, the contractive condition ( $\mathrm{Si}$ ) of Theorem 11 is satisfied. Observe that $f$ has a unique fixed point (namely, $\rho=0$ ).

Denote by $\mathscr{F}$ the class of functions $F:(0, \infty) \longrightarrow \mathbb{R}$ such that

$\left(F_{1}\right) F$ is increasing

$\left(F_{2}\right)$ For all $\alpha_{n}>0, \lim _{n \longrightarrow \infty} \alpha_{n}=0$ iff $\lim _{n \longrightarrow \infty} F\left(\alpha_{n}\right)$ $=-\infty$.

The related fixed-point Wardowski-type result for multivalued mappings in the setting of partial symmetric spaces is as follows.

Theorem 16. Let $(X, \chi)$ be a complete partial symmetric space and $f: X \longrightarrow \mathscr{C} \mathscr{B} \chi(X)$. Assume that

(i) There are $F \in \mathscr{F}$ and $\tau>0$ such that 


$$
\tau+F\left(H_{\chi}(f \rho, f \varsigma)\right) \leq F(M(\rho, \varsigma))
$$

(ii) There is $\rho_{0} \in X$ so that $\mathbb{\Im}\left(\chi, f, \rho_{0}\right)<\infty$

(iii) The function $\rho \longrightarrow \operatorname{dist}_{\chi}(\rho, f \rho)$ is lower semicontinuous.

Then, $f$ has a unique fixed point.

Proof. For such $\rho_{0} \in X$, take $\rho_{1} \in f \rho_{0}$. Define the sequence $\left\{\rho_{n}\right\} \in X$ by $\rho_{n+1} \in f \rho_{n}$. If we assume that $\rho_{n_{0}} \in f \rho_{n_{0}}$ for some $n_{0} \in \mathbb{N}$, then $\rho_{n_{0}}$ is a fixed point of $f$. Suppose $\rho_{n}$ $\notin f \rho_{n}$ for all $n \in \mathbb{N}$, so $\chi\left(\rho_{n}, \rho_{n+1}\right)>0$. Since $f$ is a generalized multivalued $F$-contraction, we have (for all $i, j \in \mathbb{N}$ )

$$
\begin{aligned}
F\left(\chi\left(\rho_{n+i}, \rho_{n+j}\right)\right) \leq & F\left(H\left(f \rho_{n-1+i}, f \rho_{n-1+j}\right)\right) \\
\leq & F\left(M\left(\rho_{n-1+i}, \rho_{n-1+j}\right)\right)-\tau \\
= & F\left(\operatorname { m a x } \left\{\chi\left(\rho_{n-1+i}, \rho_{n-1+j}\right),\right.\right. \\
& \operatorname{dist}_{\chi}\left(\rho_{n-1+i}, f \rho_{n-1+i}\right), \operatorname{dist}_{\chi}\left(\rho_{n-1+j}, f \rho_{n-1+j}\right), \\
& \left.\left.\operatorname{dist}_{\chi}\left(\rho_{n-1+i}, f \rho_{n-1+j}\right), \operatorname{dist}_{\chi}\left(\rho_{n-1+j}, f \rho_{n-1+i}\right)\right\}\right)-\tau \\
\leq & F\left(\operatorname { m a x } \left\{\chi\left(\rho_{n-1+i}, \rho_{n-1+j}\right), \chi\left(\rho_{n-1+i}, \rho_{n+i}\right),\right.\right. \\
& \left.\left.\chi\left(\rho_{n-1+j}, \rho_{n+j}\right), \chi\left(\rho_{n-1+i}, \rho_{n+j}\right), \chi\left(\rho_{n-1+j}, \rho_{n+i}\right)\right\}\right)-\tau .
\end{aligned}
$$

The above inequality is true for all $i, j \in \mathbb{N}$. Therefore, by conditions (ii) and (12), we have

$$
F\left(\mathfrak{S}\left(\chi, f, \rho_{n}\right)\right) \leq F\left(\mathfrak{S}\left(\chi, f, \rho_{n-1}\right)\right)-\tau
$$

By continuing this process, we find that

$$
F\left(\mathfrak{\Im}\left(\chi, f, \rho_{n}\right)\right) \leq F\left(\mathfrak{\Im}\left(\chi, f, \rho_{0}\right)\right)-n \tau \text {. }
$$

Now, for all $n, m \in \mathbb{N}$, we have

$$
\chi\left(\rho_{n}, \rho_{n+m}\right) \leq \mathfrak{S}\left(\chi, f, \rho_{n}\right) F\left(\mathfrak{S}\left(\chi, f, \rho_{n}\right)\right) \leq F\left(\mathfrak{S}\left(\chi, f, \rho_{0}\right)\right)-n \tau .
$$

On making the limit as $n \longrightarrow \infty$ in (45), we have

$$
\lim _{n \rightarrow \infty} F\left(\widetilde{S}\left(\chi, f, \rho_{n}\right)\right)=-\infty .
$$

Owing to conditions (ii) and $\left(F_{2}\right)$, we have

$$
\lim _{n \longrightarrow \infty} \mathfrak{S}\left(\chi, f, \rho_{n}\right)=0 \lim _{n, m \longrightarrow \infty} \chi\left(\rho_{n}, \rho_{n+m}\right)=0,
$$

so that $\left\{\rho_{n}\right\}$ is a $\chi$-Cauchy sequence in $X$. In view of the $\chi$-completeness of $X$, there is $\rho \in X$ in order that $\left\{\rho_{n}\right\} \chi$ -converges to $\rho$. Thus, we have

$$
\chi(\rho, \rho)=\lim _{n \longrightarrow \infty} \chi\left(\rho_{n}, \rho\right)=\lim _{n, m \longrightarrow \infty} \chi\left(\rho_{n}, \rho_{n+m}\right)=0 .
$$

Assume that $\rho \longrightarrow \operatorname{dist}_{\chi}(\rho, f \rho)$ is lower semicontinuous. Then, we have

$$
\operatorname{dist}_{\chi}(\rho, f \rho) \leq \liminf _{n \longrightarrow \infty} \operatorname{dist}_{\chi}\left(\rho_{n}, f \rho_{n}\right) \leq \lim _{n \longrightarrow \infty} \chi\left(\rho_{n}, \rho_{n+1}\right)=0 .
$$

Therefore, $\operatorname{dist}_{\chi}(\rho, f \rho)=0$ implies that $\rho \in f \rho$. Hence, $\rho$ is a fixed point of $f$.

For the uniqueness part, suppose there exist $\rho \neq \varsigma \in X$ such that $\rho \in f \rho$ and $\varsigma \in f \varsigma$. Thus, by the condition (i), we have

$$
\begin{aligned}
F\left(H_{\chi}(\rho, \varsigma)\right)= & F\left(H_{\chi}(f \rho, f \varsigma)\right) \leq F(M(\rho, \varsigma))-\tau \\
= & F\left(\operatorname { m a x } \left\{\chi(\rho, \varsigma), \operatorname{dist}_{\chi}(\rho, f \rho), \operatorname{dist}_{\chi}(\varsigma, f \varsigma),\right.\right. \\
& \left.\left.\operatorname{dist}_{\chi}(\rho, f \varsigma), \operatorname{dist}_{\chi}(\varsigma, f \rho)\right\}\right)-\tau \\
= & F(\max \{\chi(\rho, \varsigma), \chi(\rho, \rho), \chi(\varsigma, \varsigma), \chi(\rho, \varsigma), \chi(\varsigma, \rho)\})-\tau \\
= & F(\chi(\rho, \varsigma))-\tau,
\end{aligned}
$$

which is a contradiction. Therefore, $\chi(\rho, \varsigma)=0$, that is, $\rho=\varsigma$. It completes the proof.

Example 17. Let $X=\{0\} \cup\left\{1 / 2^{n}: n \in \mathbb{N}\right\}$ be equipped with the partial symmetric $\chi: X \times X \longrightarrow[0, \infty)$ defined by

$$
\chi(\rho, \varsigma)=\frac{1}{2}|\rho-\varsigma|^{2}+\frac{1}{4}(\max \{\rho, \varsigma\})^{2} \text { for all } \rho, \varsigma \in X .
$$

Then, $(X, \chi)$ is a $\chi$-complete symmetric space. Note that $\left\{1 / 2^{n+1}\right\}$ and $\{0\}$ are bounded sets in $(X, \chi)$. In fact

$$
\begin{aligned}
\rho & \in\left\{\frac{\overline{1}}{2^{n+1}}\right\} \Longleftrightarrow \operatorname{dist}_{\chi}\left(\rho,\left\{\frac{1}{2^{n+1}}\right\}\right) \\
& =\chi(\rho, \rho) \Longleftrightarrow \min \left\{\frac{1}{2}\left|\rho-\frac{1}{2^{n+1}}\right|^{2}+\frac{1}{4}\left(\max \left\{\rho, \frac{1}{2^{n+1}}\right\}\right)^{2}\right\} \\
& =\frac{\rho^{2}}{4} \Longleftrightarrow \rho=\frac{1}{2^{n+1}} \Longleftrightarrow \rho \in\left\{\frac{1}{2^{n+1}}\right\} .
\end{aligned}
$$

Hence, $\left\{1 / 2^{n+1}\right\}$ is closed. Next

$\rho \in\{\overline{0}\} \Longleftrightarrow \operatorname{dist}_{\chi}(\rho,\{0\})=\chi(\rho, \rho) \Longleftrightarrow \frac{3 \rho^{2}}{4}=\frac{\rho^{2}}{4} \Longleftrightarrow \rho \in\{0\}$.

Hence, $\{0\}$ is also closed with respect to the partial symmetric $\chi$.

Define $f: X \longrightarrow \mathscr{C} \mathscr{B}^{\chi}(X)$ and $F:(0, \infty) \longrightarrow \mathbb{R}$ by

$$
f \rho=\left(\begin{array}{ll}
\left\{\frac{1}{2^{n+1}}\right\}, & \text { if } \rho=\frac{1}{2^{n}} \\
\{0\}, & \text { if } \rho=0,
\end{array}\right.
$$

and $F(\alpha)=\ln (\alpha)$ for $\alpha>0$, respectively. Then 


$$
\operatorname{dist}_{\chi}(\rho, f \rho)=\left(\begin{array}{ll}
\frac{3}{2}\left(\frac{1}{2^{n+1}}\right)^{2}, & \text { if } \rho=\frac{1}{2^{n}} \\
0, & \text { if } \rho=0 .
\end{array}\right.
$$

Hence, $\rho \longrightarrow \operatorname{dist}_{\chi}(\rho, f \rho)$ is lower semicontinuous. Now, we will show that the contractive condition (i) of Theorem 16 is satisfied.

Case 18. Let $\rho, \varsigma \in\left\{1 / 2^{n}\right\}$ (say $\rho=1 / 2^{n_{1}}$ and $\rho=1 / 2^{n_{2}}$ for all $\left.n_{1}, n_{2} \in \mathbb{N}\right)$. Here

$$
\begin{aligned}
H_{\chi}(f \rho, f \varsigma) & =H_{\chi}\left(\left\{\frac{1}{2^{n_{1}+1}}\right\},\left\{\frac{1}{2^{n_{2}+1}}\right\}\right) \\
& =\frac{1}{2}\left|\frac{1}{2^{n_{1}+1}}-\frac{1}{2^{n_{2}+1}}\right|^{2}+\frac{1}{4}\left(\max \left\{\frac{1}{2^{n_{1}+1}}, \frac{1}{2^{n_{2}+1}}\right\}\right)^{2} \\
& =\frac{1}{4}\left(\frac{1}{2}\left|\frac{1}{2^{n_{1}}}-\frac{1}{2^{n_{2}}}\right|^{2}+\frac{1}{4}\left(\max \left\{\frac{1}{2^{n_{1}}}, \frac{1}{2^{n_{2}}}\right\}\right)^{2}\right) \\
& =\frac{1}{4} \chi(\rho, \varsigma) \leq \frac{1}{4} M(\rho, \varsigma) .
\end{aligned}
$$

That is (for $\tau=\ln (4)$ )

$$
\tau+F\left(H_{\chi}(f \rho, f \varsigma)\right) \leq F(M(\rho, \varsigma))
$$

Case 19. Let $\rho \in\left\{1 / 2^{n}\right\}$ and $\varsigma \in\{0\}$. Then

$$
\begin{aligned}
H_{\chi}(f \rho, f \varsigma) & =H_{\chi}\left(\left\{\frac{1}{2^{n+1}}\right\},\{0\}\right) \\
& =\max \left\{\delta_{\chi}\left(\left\{\frac{1}{2^{n+1}}\right\},\{0\}\right), \delta_{\chi}\left(\{0\},\left\{\frac{1}{2^{n+1}}\right\}\right)\right\} \\
& =\frac{3}{4}\left(\frac{1}{2^{n+1}}\right)^{2} .
\end{aligned}
$$

Also

$$
\begin{aligned}
M\left(\frac{1}{2^{n}}, 0\right)= & \max \left\{\chi\left(\frac{1}{2^{n}}, 0\right), \operatorname{dist}_{\chi}\left(\frac{1}{2^{n}}, f \frac{1}{2^{n}}\right), \operatorname{dist}_{\chi}(0,\{0\}),\right. \\
& \left.\operatorname{dist}_{\chi}\left(\frac{1}{2^{n}},\{0\}\right) \operatorname{dist}_{\chi}\left(0, \frac{1}{2^{n+1}}\right)\right\} \\
= & \max \left\{\frac{3}{4}\left(\frac{1}{2^{n}}\right)^{2}, \frac{3}{8}\left(\frac{1}{2^{n}}\right)^{2}, 0, \frac{3}{4}\left(\frac{1}{2^{n}}\right)^{2}, \frac{3}{16}\left(\frac{1}{2^{n}}\right)^{2}\right\} \\
= & \frac{3}{4}\left(\frac{1}{2^{n}}\right)^{2} .
\end{aligned}
$$

For $\tau=\ln (4)$, one writes

$$
\tau+F\left(H_{\chi}(f \rho, f \varsigma)\right) \leq F(M(\rho, \varsigma)) .
$$

Therefore, all the conditions of Theorem 16 are satisfied, and $f$ has a unique fixed point (namely, $\varsigma=0$ ).

\section{An Application to an Integral Inclusion}

Let $X=C([a, b], \mathbb{R})$ be the set of all continuous real valued functions defined on $[a, b]$. Now, we consider the following integral inclusion of Volterra type:

$$
\rho(t) \in \int_{a}^{t} G(t, s, \rho(s)) d s+h(t), \forall t \in[a, b] \text { and } h \in X,
$$

where $G:[a, b] \times[a, b] \times \mathbb{R} \longrightarrow K_{c v}(\mathbb{R})$ is a multivalued operator and $K_{c v}(\mathbb{R})$ is the set of nonempty compact and convex subsets of $\mathbb{R}$.

Define $\chi: X \times X \longrightarrow \mathbb{R}^{+}$by

$\chi(\rho(t), \varsigma(t))=\sup _{t \in[a, b]}|\rho(t)-\varsigma(t)|^{p}+\sup _{t \in[a, b]}|\rho(t)-\varsigma(t)|^{q}, \quad q>p>1$.

Then, $(X, \chi)$ is a $\chi$-complete partial symmetric space.

Now, we are ready to present our result as follows:

Theorem 20. Suppose that for all $\rho, \varsigma \in C([a, b], \mathbb{R})$ there exist a continuous function $j: X \longrightarrow \mathbb{R}$ and $\int_{a}^{t} j(s) d s \leq\left(e^{-\tau}\right)^{1 / p}$ with $\tau>0$ such that

$$
H_{\chi}(G(t, s, \rho(s)), G(t, s, \varsigma(s))) \leq j(s)|\rho(s)-\varsigma(s)|, \forall t, s \in[a, b] .
$$

Then, the integral inclusion (61) has a unique solution.

Proof. Let us define the multivalued operator $f: X \longrightarrow \mathscr{C}$ $\mathscr{B}^{\chi}(X)$ by $f \rho(t)=\in X:\left\{v \in \int_{0}^{1} G(t, s, \rho(s)) d s+h(t)\right.$, for all $t$ $\in[0,1]$ and $h \in X\}$.

Let $\rho \in X$ and consider $G_{\rho}=G(t, s, \rho(s)), t, s \in[a, b]$. For a multivalued operator $G_{\rho}:[a, b] \times[a, b] \longrightarrow K_{c v}(\mathbb{R})$, there exists a continuous operator $g_{\rho}:[a, b] \times[a, b] \longrightarrow \mathbb{R}$ (by Michael's selection theorem) such that $g_{\rho}(t, s) \in G_{\rho}(t, s)$, for all $t, s \in[a, b]$. It implies that $\int_{a}^{t} g_{\rho}(t, s) d s+h(t) \in f \rho(t)$. Thus, the operator $f \rho$ is nonempty. Note that $f \rho$ is closed. For more details, see [22].

Firstly, we check the condition (i) of Theorem 16. Let $\rho, \varsigma$ $\in X$ be such that $u \in f \rho$. Then, there exists $g_{\rho}(t, s) \in G_{\rho}(t, s)$, for all $t, s \in[a, b]$ such that $u(t)=\int_{a}^{t} g_{\rho}(t, s) d s+h(t), t \in[a, b]$.

Besides, for all $\rho, \varsigma \in X$, we have

$$
H_{\chi}(G(t, s, \rho(s)), G(t, s, \varsigma(s))) \leq j(s)|\rho(s)-\varsigma(s)|, \forall t, s \in[a, b] .
$$

Consequently, there exists $v(t, s) \in G_{\zeta}(t, s)$ such that

$$
\left|g_{\rho}(t, s)-v(t, s)\right| \leq j(s)|\rho(s)-\varsigma(s)|, \forall t, s \in[a, b] .
$$


Let us consider the multivalued operator $S$ defined by

$$
\begin{aligned}
S(t, s) & =G_{\zeta}(t, s) \cap\left\{w \in \mathbb{R}:\left|g_{\rho}(t, s)-w\right|\right. \\
& \leq j(s)|\rho(s)-\varsigma(s)|, \forall t, s \in[a, b]\} .
\end{aligned}
$$

Since $f$ is a lower semicontinuous, it follows that there exists $g_{\varsigma}:[a, b] \times[a, b] \longrightarrow \mathbb{R}$ such that $g_{\varsigma}(t, s) \in S(t, s)$, for all $t, s \in[a, b]$. Then, we have

$$
\begin{aligned}
& z(t)=\int_{a}^{t} g_{\varsigma}(t, s) d s+h(t) \in \int_{a}^{t} G(t, s, \varsigma(s)) d s+h(t), t \in[a, b], \\
& |v(t)-z(t)|^{p}+|v(t)-z(t)|^{q}=\left(\int_{a}^{t}\left|g_{\rho}(t, s)-g_{c}(t, s)\right| d s\right)^{p} \\
& +\left(\int_{a}^{t}\left|g_{\rho}(t, s)-g_{\varsigma}(t, s)\right| d s\right)^{q} \\
& \leq\left(\int_{a}^{t} j(s)|\rho(s)-\varsigma(s)| d s\right)^{p}+\left(\int_{a}^{t} j(s)|\rho(s)-\varsigma(s)| d s\right)^{q} \\
& \leq\left(\sqrt[p]{\sup _{t \in[a, b]}|\rho(t)-\varsigma(t)|^{p}} \int_{a}^{t} j(s) d s\right)^{p}+\left(\sqrt[q]{\sup _{t \in[a, b]}|\rho(t)-\varsigma(t)|^{q}} \int_{a}^{t} j(s) d s\right)^{q} \\
& =\sup _{t \in[a, b]}|\rho(t)-\varsigma(t)|^{p}\left(\int_{a}^{t} j(s) d s\right)^{p}+\sup _{t \in[a, b]}|\rho(t)-\varsigma(t)|^{q}\left(\int_{a}^{t} j(s) d s\right)^{q} \\
& \leq \sup _{t \in[a, b]}|\rho(t)-\varsigma(t)|^{p} e^{-\tau}+\sup _{t \in[a, b]}|\rho(t)-\varsigma(t)|^{q}\left(e^{-\tau}\right)^{q / p} \\
& \leq\left(\sup _{t \in[a, b]}|\rho(t)-\varsigma(t)|^{p}+\sup _{t \in[a, b]}|\rho(t)-\varsigma(t)|^{q}\right) e^{-\tau}(\text { since, } q>p) \\
& =\chi(\rho, \varsigma) e^{-\tau} \text {. }
\end{aligned}
$$

Hence, we have

$$
\chi(\nu, z) \leq e^{-\tau} \chi(\rho, \varsigma) .
$$

On interchanging the job of $\rho$ and $\varsigma$, we have

$$
H_{\chi}(f \rho, f \varsigma) \leq e^{-\tau} \chi(\rho, \varsigma)
$$

By using $F(\alpha)=\ln (\alpha)$, we have

$$
\tau+F\left(H_{\chi}(f \rho, f \varsigma)\right) \leq \chi(\rho, \varsigma) \leq F(M(\rho, \varsigma))
$$

Thus, all the hypotheses of Theorem 16 are satisfied. Hence, the operator $f$ has exactly one fixed point, that is, the Volterra integral inclusion (61) has a unique solution.

Remark 21. Consider the following differential inclusion (for $\rho \in C([a, b], \mathbb{R}))$ :

$$
\rho^{\prime}(t) \in G(t, s, \rho(s)), t, s \in[a, b]
$$

where $G:[a, b] \times[a, b] \times \mathbb{R} \longrightarrow K_{c v}(\mathbb{R})$ is a multivalued operator. Observe that, for $h(t)=0,(71)$ is equivalent to (61). Also, G satisfies the condition (63) of Theorem20. Then, applying Theorem20, the differential inclusion (71) has a unique solution.
Example 22. For all $\rho, \varsigma \in C([a, b], \mathbb{R})$, let

$$
G(t, s, \rho(s))=\left\{v \in \mathbb{R}: g_{1}(t, s, \rho(s)) \leq v \leq g_{2}(t, s, \rho(s))\right\},
$$

where $g_{1}(t, s, \rho(s))$ (resp., $g_{2}(t, s, \rho(s))$ ) is upper semicontinuous (resp., lower semicontinuous) on $[a, b] \times[a, b]$. For $\rho \in C$ $([a, b], \mathbb{R})$, assume the following:

$$
\rho^{\prime}(t) \in G(t, s, \rho(s)), t, s \in[a, b]
$$

For all $\rho, \varsigma \in C([a, b], \mathbb{R})$, there exist a continuous function $j: X \longrightarrow \mathbb{R}$ and $\tau>0$ such that $\int_{a}^{t} j(s) d s \leq\left(e^{-\tau}\right)^{1 / p}$ and

$$
\begin{aligned}
& \max \left|g_{1}(t, s, \rho(s))-g_{2}(t, s, \varsigma(s))\right| \\
& \leq j(s)|\rho(s)-\varsigma(s)|, \text { for all } t, s \in[a, b]
\end{aligned}
$$

Hence, $G$ is a compact and convex valued operator. Thus, $G$ satisfies the condition (63) of Theorem 20 (for $h(t)=0)$, so (74) has a unique solution.

\section{Conclusion}

We are concerned with Suzuki and Wardowski-type contraction multivalued mappings in the setting of partial symmetric spaces. We also studied a system of integral inclusions. It would be interesting to work on more generalized contraction mappings involving simulation or control functions.

\section{Data Availability}

The data used to support the findings of this study are available from the corresponding author upon request.

\section{Conflicts of Interest}

The authors declare that they have no competing interests.

\section{Authors' Contributions}

All authors contributed equally and significantly in writing this article. All authors read and approved the final manuscript.

\section{References}

[1] S. Banach, "Sur les opérations dans les ensembles abstraits et leur application aux équations intégrales," Fundamenta Mathematicae, vol. 3, pp. 133-181, 1922.

[2] S. G. Matthews, "Partial metric topology," Annals of the New York Academy of Sciences, vol. 728, no. 1 General Topol, pp. 183-197, 1994.

[3] M. Asim and M. Imdad, "Partial JS-metric spaces and fixed fixed point results," Indian Journal of Mathematics, vol. 61, no. 2, pp. 175-186, 2019.

[4] A. Amini-Harandi, "Metric-like spaces, partial metric spaces and fixed points," Fixed Point Theory and Applications, vol. 2012, no. 1, Article ID 204, 2012. 
[5] S. Czerwik, "Contraction mappings in b-metric spaces," Acta Mathematica et Informatica Universitatis Ostraviensis, vol. 1, no. 1, pp. 5-11, 1993.

[6] A. Branciari, "A fixed point theorem of Banach-Caccioppoli type on a class of generalized metric spaces," Publicationes Mathematiques, vol. 57, pp. 31-37, 2000.

[7] N. Mlaiki, H. Aydi, N. Souayah, and T. Abdeljawad, "Controlled metric type spaces and the related contraction principle," Mathematics, vol. 6, no. 10, p. 194, 2018.

[8] T. Abdeljawad, N. Mlaiki, H. Aydi, and N. Souayah, "Double controlled metric type spaces and some fixed point results," Mathematics, vol. 6, no. 12, p. 320, 2018.

[9] E. Karapinar, S. Czerwik, and H. Aydi, “ $(\alpha, \psi)$-Meir-Keeler contraction mappings in generalized b-metric spaces," Journal of Function spaces, vol. 2018, Article ID 3264620, 4 pages, 2018.

[10] M. Asim, M. Imdad, and S. Radenović, "Fixed point results in extended rectangular $b$-metric spaces with an application," U.P.B. Sci. Bull., Series A, vol. 81, no. 2, pp. 11-20, 2019.

[11] M. Asim, A. R. Khan, and M. Imdad, "Fixed point results in partial symmetric spaces with an application," Axioms, vol. 8, no. 1, p. 13, 2019.

[12] H. Aydi, M. Abbas, and C. Vetro, "Partial Hausdorff metric and Nadler's fixed point theorem on partial metric spaces," Topology and its Applications, vol. 159, no. 14, pp. 32343242, 2012.

[13] E. Ameer, H. Aydi, M. Arshad, H. Alsamir, and M. S. Noorani, "Hybrid multivalued type contraction mappings in $\alpha_{K}$-complete partial b-metric spaces and applications," Symmetry, vol. 11, no. 1, p. 86, 2019.

[14] S. B. Nadler, "Multi-valued contraction mappings," Pacific Journal of Mathematics, vol. 30, no. 2, pp. 475-488, 1969.

[15] T. Suzuki, "A generalized Banach contraction principle that characterizes metric completeness," Proceedings of the American Mathematical Society, vol. 136, pp. 1861-1870, 2007.

[16] D. Wardowski, "Fixed points of a new type of contractive mappings in complete metric spaces," Fixed Point Theory and Applications, vol. 2012, 2012.

[17] D. Wardowski and N. Van Dung, "Fixed points of F-weak contractions on complete metric spaces," Demonstratio Mathematica, vol. 47, no. 1, pp. 146-155, 2014.

[18] V. Parvaneh, N. Hussain, and Z. Kadelburg, "Generalized Wardowski type fixed point theorems via $\alpha$ admissible FG-contractions in b-metric spaces," Acta Mathematica Scientia, vol. 36, no. 5, pp. 1445-1456, 2016.

[19] H. Isik, V. Parvaneh, B. Mohammadi, and I. Altun, "Common fixed point results for generalized Wardowski type contractive multi-valued mappings," Mathematics, vol. 7, no. 11, article 1130, 2019.

[20] E. Ameer, H. Aydi, M. Arshad, and M. De la Sen, "Hybrid Ćirić type graphic $\Upsilon, \Lambda$-contraction mappings with applications to electric circuit and fractional differential equations," Symmetry, vol. 12, no. 3, p. 467, 2020.

[21] P. Patle, D. Patel, H. Aydi, and S. Radenovic, "On $H^{+}$-type multivalued contractions and applications in symmetric and probabilistic spaces," Mathematics, vol. 7, no. 2, p. 144, 2019.

[22] A. Sîntămărian, "Integral inclusions of Fredholm type relative to multivalued $\phi$-contraction," Seminar on Fixed Point Theory Cluj-Napoca, vol. 3, pp. 361-368, 2002. 